\title{
Maternal \& perinatal outcome in hypertensive disorders of pregnancy in a tertiary care hospital in northern india
}

\begin{abstract}
Background: Hypertension is the most common medical disorder encountered during pregnancy, occurring in $6-8 \%$ of pregnancies. Women with hypertensive disorders of pregnancy (HDP) are all at increased risk of complications antenatally and in the puerperium.

Objective: This study aimed to determine the maternal and perinatal outcomes of hospitalized pregnant cases with hypertension.

Methods: This was a 3 years retrospective study conducted in a tertiary care centre of Northern India from July 2011 to June 2014. Maternal and Perinatal outcomes were compared among the HDP groups and results were analysed and tabulated by SPSS version, using chi square method.

Results: Out of 2,989 deliveries during the defined period, the incidence of women presenting with HDP was $6.92 \%(\mathrm{n}=207)$. Of these $50.2 \%(104 / 207)$ were pre eclampsia, $35.7 \%(74 / 207)$ eclampsia, $12.5 \%$ (26/207) were gestational hypertension and rest were chronic hypertension. The mean age of women presenting with HDP was $25.42 \pm 4.64$ years. Co-morbid conditions like Gestational diabetes mellitus (GDM) was seen in 1.4\% (3/207), twin pregnancy was seen in 5.79\% (12/207) and obesity was seen in $7.24 \%(15 / 207)$ cases of HDP. There were 2 maternal deaths and 7 neonatal deaths. Maternal complications like acute renal failure (ARF), congestive heart failure (CHF), acute respiratory distress syndrome (ARDS) \& disseminated intravascular coagulation (DIC) were seen more commonly in severe pre-eclampsia group. $62.8 \%$ babies (130/207) were premature. Poor neonatal outcome was higher in eclampsia group followed by severe pre-eclampsia group.

Conclusion: A substantial burden of maternal and perinatal morbidity and mortality is associated with hypertensive disorders of pregnancy, more so in eclampsia and severe preeclampsia group as compared to gestational hypertension and mild pre-eclampsia.
\end{abstract}

Keywords: Hypertension, Maternal outcome, Foetal outcome, Low birth weight, Eclampsia, Severe pre-eclampsia
Volume 6 Issue 6 - 2017

\section{Charu Sharma, Smriti Gupta, Mamta Tyagi,} Poonam Mani, Jasneet Dhingra, Renu Rana Department of Obstetrics \& Gynaecology, SN Medical College, India

Correspondence: Charu Sharma,Assistant Professor, Department of Obstetrics \& Gynaecology, SN Medical College, Jodhpur, Rajasthan, India, Tel 9 I-70639562I5, Emailsharma.charu65।@gmail.com

Received: April 17, 2017| Published: May 04, 2017
Abbreviations: GH, Gestational Hypertension; CH, Chronic Hypertension; PE, Pre Eclampsia

\section{Introduction}

Hypertensive disorders are the most common medical disorders encountered during pregnancy, occurring in approximately $7 \%$ to $10 \%$ of all pregnancies. ${ }^{1}$ They are associated with significant maternal and perinatal morbidity and mortality and have a wide spectrum of presentation, ranging from minimal elevation of blood pressure to severe hypertension with multiple organ dysfunctions. Among the hypertensive disorders, the pre-eclampsia syndrome, either alone or superimposed on chronic hypertension, is the most dangerous. Eclampsia is the convulsive form of pre-eclampsia and affects $0.1 \%$ of all the pregnancies. ${ }^{2} \mathrm{New}$ onset non proteinuric hypertension during pregnancy, termed gestational hypertension, is followed by signs and symptoms of pre-eclampsia almost half the time. Women who develop pre-eclampsia in pregnancy are at greater risk of cardiovascular and cerebrovascular events even years after their pregnancies. Hypertension complicating pregnancy is a major cause of preterm births resulting in perinatal deaths of foetuses. In low income and middle income countries, pre-eclampsia and eclampsia are associated with $10-15 \%$ of direct maternal deaths. WHO estimates the incidence of preeclampsia to be seven times higher in developing countries ( $2.8 \%$ of live births) than in developed countries $(0.4 \%) .^{3}$ Incidence of eclampsia in developing nations varies widely, ranging from 1 case per 100 pregnancies to 1 case per 3448 pregnancies. For patients obtaining prenatal care, the incidence is about 1 in 800 patients. ${ }^{1}$ The incidence of maternal mortality due to HDP is estimated at 20$33 \% .{ }^{4}$ Prevention of any disease process requires the availability of methods for prediction of those at high risk for the disorder. Although numerous clinical and biochemical tests have been proposed for prediction or early detection of preeclampsia, most remain unrealistic for general use in most developing countries. At present, there is not a single reliable and cost-effective screening test for preeclampsia which can be recommended for use in most developing countries. ${ }^{5}$ Although some studies on uterine artery Doppler and first-trimester maternal serum markers for early detection of preeclampsia have shown promise, there is not enough evidence to suggest their routine use in clinical practice, more so in resource poor settings. ${ }^{6-8}$

This study was conducted to evaluate the foeto-maternal outcome in women with hypertensive disorders of pregnancy who delivered in our hospital. Although many such studies have been published earlier and our study is just an addition to the existing literature but for us it helped to set up a protocol to prevent such untoward consequences. 


\section{Materials and methods}

This was a hospital based study done in Subharti Medical College, Meerut, by evaluating the records of women who had delivered in our hospital from July 2011 to June 2014. All women who presented with hypertensive disorders of pregnancy and delivered in the hospital and whose records were complete, were included in the study and divided into 5 groups namely, Gestational hypertension (GH), Mild pre-eclampsia (PE), Severe pre-eclampsia, Eclampsia and Chronic hypertension with superimposed pre-eclampsia (CHPE) based on their clinical presentation at admission. After excluding all incomplete data entries, the sample size was finalised at 207.

Chronic hypertension was defined as hypertension diagnosed before pregnancy and / or diastolic pressure $\geq 90 \mathrm{mmHg}$ and / or antihypertensive medications started each before the 20 weeks of gestation uncomplicated by de novo proteinuria; Gestational hypertension was defined as hypertension with systolic blood pressure $\geq 140 \mathrm{mmHg}$ and/or diastolic pressure $\geq 90 \mathrm{mmHg}$ for the first time after 20 weeks of gestation and/or in women hospitalized during pregnancy because of hypertension and/or if antihypertensive medications prescribed for the first time after 20 weeks of pregnancy without proteinuria; Preeclampsia: women different from gestational hypertension because of development of de novo proteinuria $(\geq 0.3 \mathrm{~g} / 24 \mathrm{~h})$. Preeclampsia superimposed on chronic hypertension: the criterion for chronic hypertension is met along with the criteria for preeclampsia. ${ }^{9}$ Preeclampsia was further classified into mild and severe. Cut offs used to define severe are BP of $160 / 110 \mathrm{mmHg}$ or higher along with symptoms like cerebral or visual disturbances, severe and persistent epigastric pain or right upper quadrant pain, pulmonary oedema, thrombocytopenia and fetal growth restriction. ${ }^{10}$ Gestational age (GA) was defined on the basis of last maternal menstrual period and confirmed by early ultrasound examination; patients with uncertain GA were not included. LBW was defined as a birth weight (BW) below 2500 gm, very low birth weight (VLBW) was defined as BW below 1500g. A small for gestational age (SGA) was defined as a newborn infant with a birth weight below the 10th percentile according to the national standard curve for singleton birth. The research was approved by the institutional ethical committee of the Subharti University.

Information regarding the patient's age, parity and associated medical illness was noted and fed into Microsoft Excel sheet. The details of the period of gestation at which pregnancy was terminated and foeto-maternal outcomes were also collected. The data was then tabulated and analysed using Chi square test. The differences between the groups were considered significant if the $p$ value was less than 0.05 .

\section{Results}

In this study, records of 2,989 women who delivered in our tertiary hospital were reviewed and of these, 256 women had hypertensive disorders of pregnancy. Forty nine of these women had either left the hospital against medical advice or their records were incomplete so their outcome could not be followed and hence were excluded from the study. Remaining 207 women were included and analysed. The incidence of HDP came out to be $6.92 \%$. Of these, $12.5 \%(26 / 207)$ women had gestational hypertension, $13.5 \%$ (28/207) women had mild pre-eclampsia, $36.7 \%(76 / 207)$ had severe pre-eclampsia and $35.7 \%(74 / 207)$ women presented with eclampsia (Figure 1). Only three women had preeclampsia superimposed on chronic hypertension and hence this group was not included for statistical analysis and calculation of $\mathrm{p}$ value.

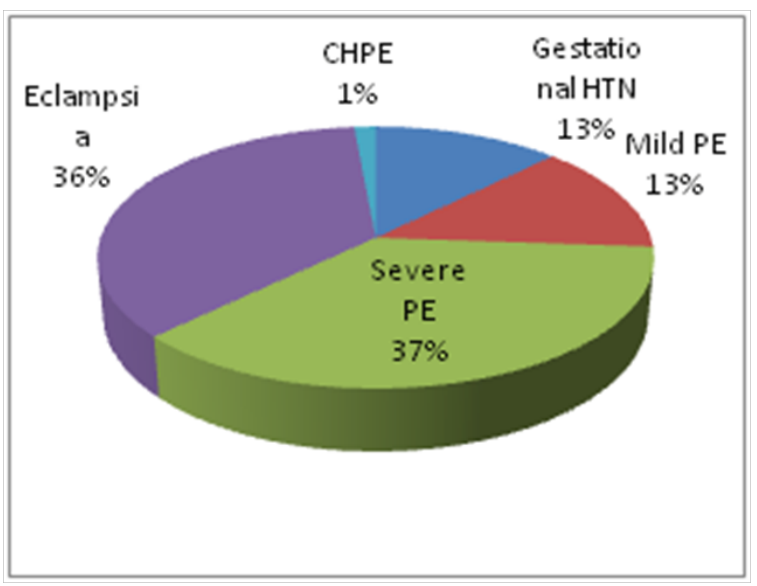

Figure I Frequency of Hypertensive disorders of pregnancy.

The mean age of cases presenting with HDP was $25.42 \pm 4.64$ years. Although majority of the women with HDP (70.5\%) belonged to the age group of 18 to 27 years. This difference in the distribution according to age within the four groups of hypertensive disorders of pregnancy was statistically insignificant $(\mathrm{p}=0.064)$. The hypertensive disorders were more common among multigravida (53.1\%). However, on comparing the four groups of hypertension, the difference in parity was not statistically significant $(\mathrm{p}=0.154)$ (Table 1). Prematurity was seen in $62.8 \%(130 / 207)$ babies. Most of the preterm deliveries were in the eclampsia group, followed by severe pre-eclampsia and gestational hypertension group. Most of the women in our study, who had gestational hypertension or mild pre-eclampsia, carried their pregnancy till term. This difference was statistically highly significant with $\mathrm{p}$ value of 0.007 .

Table I Demographic characteristics of women with HDP

\begin{tabular}{ll}
\hline Demographic characteristics & Frequency (\%) $\mathbf{n = 2 0 7}$ \\
\hline Age in years & $146(70.5)$ \\
I $8-27$ & $55(26.5)$ \\
$28-37$ & $6(2.8)$ \\
$38-47$ & \\
Parity & $97(46.8)$ \\
Primi gravida & $\mathrm{I} 10(53.1)$ \\
Multigravida & \\
Mode of delivery & $\mathrm{I} 12(54.1)$ \\
Vaginal & $95(45.8)$ \\
Caesarean & \\
Gestational age & $\mathrm{I} 30(62.8)$ \\
Preterm(<37weeks) & $77(37.1)$ \\
Term (>37 weeks) & \\
Birth weight & $47(22.7)$ \\
VLBW (<I500 gms) & $102(49.2)$ \\
LBW (<2500 gms) &
\end{tabular}

Of the total deliveries in HDP group, 112 women had vaginal delivery and 95 women underwent caesarean section. The most common indication of caesarean delivery was foetal distress. The difference in the number of women who had vaginal deliveries and caesarean deliveries was statistically insignificant with $p$ value of 0.622 (Table 1).

On analysing the maternal outcome in these women (Table 2), $81.6 \%(169 / 207)$ reported cases presented no complication. However, significant morbidity was noted in $17.3 \%(36 / 207)$ cases. There 
were 2 maternal deaths, one in severe pre eclampsia and another in eclampsia group, both probably due to intracerebral haemorrhage. All these maternal complications were observed only in the two groups of severe pre-eclampsia and eclampsia. None of the women with gestational hypertension or mild pre eclampsia had complications. This difference was statistically significant with $p$ value of 0.001 .

We found that anaemia was the most commonly associated medical disorder in these women (16.4\%). Three women had GDM (Gestational diabetes mellitus) with HDP, of which one presented with gestational hypertension and two developed severe pre-eclampsia in last trimester. Twelve women had twin pregnancies and two had rheumatic heart disease.

In total, 218 babies were delivered of 207 women including the twins. Of these 188 were live births and 30 were stillbirths. Neonatal intensive care unit (NICU) admission was observed in 41.2\% (90/218) babies and of these 15 required ventilator support. There were seven early neonatal deaths. On comparing the four groups, we found that poor neonatal outcome in terms of low APGAR, IUGR, LBW, higher NICU admissions and need for ventilator for baby was much higher in the women with eclampsia and severe pre-eclampsia. The Table 3 also depicts that neonatal outcome was better in gestational hypertension and mild pre-eclampsia group. This difference was highly significant with $\mathrm{p}$ value of $<0.001$. Forty seven babies had birth weight less than $1.5 \mathrm{~kg}$ even though only five babies were born before 28 weeks of gestation. Of these, most of the babies were born to mothers with severe pre- eclampsia or eclampsia (Table 3 ). The p value was 0.002 .

Table 2 Maternal outcome in various groups of HDP $(n=207)$

\begin{tabular}{|c|c|c|c|c|c|c|}
\hline Maternal outcome & GH $(n I=26)$ & Mild PE (n2 = 28) & Severe PE $(n 3=76)$ & Eclampsia (n4=74) & CHPE $(n 5=3)$ & Total no. (\%) \\
\hline Normal & 26 & 28 & $59(28.5)$ & $56(27.0)$ & 3 & $169(81.6)$ \\
\hline \multicolumn{7}{|l|}{ Morbidity } \\
\hline ICU adm & 0 & 0 & $7(3.3)$ & $5(2.4)$ & 0 & $12(5.7)$ \\
\hline Ventilator & 0 & 0 & $2(0.9)$ & $49(1.9)$ & 0 & $6(2.8)$ \\
\hline Mortality & 0 & 0 & I (0.4) & $\mathrm{I}(0.4)$ & 0 & $2(0.9)$ \\
\hline ARF & 0 & 0 & $3(1.4)$ & $2(0.9)$ & 0 & $5(2.4)$ \\
\hline DIC / HELLP & 0 & 0 & $3(1.4)$ & $2(0.9)$ & 0 & $5(2.4)$ \\
\hline PPH & 0 & 0 & $10(4.8)$ & $12(5.7)$ & 0 & I (0.4) \\
\hline $\mathrm{CHF}$ & 0 & 0 & $3(1.4)$ & $2(0.9)$ & 0 & $3(1.4)$ \\
\hline Intra cerebral haemorrhage & 0 & 0 & $\mathrm{I}(0.4)$ & I (0.4) & 0 & $2(0.9)$ \\
\hline ARDS & 0 & 0 & $2(0.9)$ & I (0.4) & 0 & $3(1.4)$ \\
\hline RD2 & 0 & 0 & 0 & I (0.4) & 0 & I (0.4) \\
\hline
\end{tabular}

Table 3 Perinatal outcome in various groups of HDP ( $n=218$ babies)

\begin{tabular}{lllllll}
\hline Fetal outcome & GH & Mild PE & Severe PE & Eclampsia & CHPE & Total no. (\%) \\
\hline Normal & 12 & 14 & 22 & 9 & 3 & $60(27.5)$ \\
IUD/Still Birth & 1 & 2 & 15 & 12 & 0 & $30(13.7)$ \\
NICU adm. & 9 & 7 & 28 & 46 & 0 & $90(41.2)$ \\
Ventilator & 1 & 0 & 3 & 11 & 0 & $15(6.8)$ \\
Neonatal Death & 0 & 0 & 2 & 5 & 0 & $07(3.2)$ \\
Low APGAR & 4 & 7 & 17 & 39 & 0 & $67(30.7)$ \\
IUGR & II & 5 & 34 & 40 & 0 & $90(41.2)$ \\
\hline
\end{tabular}

\section{Discussion}

Hypertensive disorders of pregnancy are considered to be a major worldwide health problem causing an increased risk of perinatal and maternal morbidity and mortality. Many theories regarding its etiology have been suggested including abnormal placentation, immunologic phenomenon, coagulation abnormalities, angiogenesis factors or endothelial damage. ${ }^{1}$

The prevalence of hypertensive disorder of pregnancy is different according to the geographic regions of the world and ranges from $1.5 \%$ in Sweden to 7.5\% in Brazil. In India the prevalence of HDP has been reported to be $6-8 \% .{ }^{9}$ These differences can be due to racial reasons, socioeconomic status and some other demographic parameters such as age and parity. Moreover, some centres serve as a referral medical facility for an extended number of primary health care units of the surrounding rural areas. The incidence of HDP in our study was $6.92 \%$. Out of total 2,989 deliveries, 104 deliveries were found to be complicated by pre-eclampsia. Hence the overall incidence of pre-eclampsia in our study was $3.47 \%$ and that of eclampsia $2.47 \%$. Prakash et al. ${ }^{10}$ reported the incidence of pre-eclampsia to be $5.8 \% .{ }^{10}$
The extreme ages of reproductive years are well-known risk factors for hypertension during pregnancy with high incidence rates in teenagers. Many authors have identified young age as a risk factor for hypertension during pregnancy ${ }^{11,12}$ as is the case in the present study. However in another systematic review, it was observed that a maternal age over 40 roughly doubles the risk of pre eclampsia in both primiparous \& multiparous women. A similar result was observed by Assis et al. ${ }^{13}$ who found that age above 30 years was associated with a risk for preeclampsia super-imposed on chronic hypertension (OR: 5.218; 95\% CI: 1.873 to 14.536 ).

Nulliparity is widely reported as a risk factor for hypertensive disorders in pregnancy. ${ }^{13-15}$ But in the present study, we found that multiparity was more associated with HDP as compared with nulliparity, however the difference was not statistically significant. Our findings did not match with the aforementioned literature in this regard.

Hypertensive disorders of pregnancy are responsible for significant maternal and perinatal morbidity and mortality. Gestational hypertension and chronic hypertension cases may have superimposed pre eclampsia; mild pre eclampsia may turn into severe pre eclampsia 
which may further get complicated by eclampsia. Moreover many other clinical conditions may mimic pre eclampsia. So it is always better to have a list of differential diagnosis with deadly acute fatty liver of pregnancy (AFLP), being one of them, in mind, whenever dealing with complicated HDP.

Devi \& Uday $^{16}$ in 2001 drew attention to some of the unusual accompaniments of HDP. In their study, the incidence of HDP was $11.7 \%$ which was much higher than that in our study. ${ }^{16}$ Ascites was observed in $2.8 \%$, pleural effusion in $1.2 \%$, HELLP syndrome in $0.8 \%$, cortical venous thrombosis in $2.8 \%$ and duodenal perforation in one patient. ${ }^{16}$ Prakash et al. ${ }^{10}$ reported HELLP syndrome in $7.5 \%$ of cases of pre eclampsia whereas Sachan et al. ${ }^{17}$ in 2013 reported it to be $3.07 \% .^{10-17}$ All these additional features worsen the pregnancy outcome and are required to be watched for meticulously. The hazards of convulsions in pregnancy have been documented for centuries. Eclamptic convulsions become a life threatening emergency when further complicated by abruptio placentae, pulmonary oedema, intracerebral haemorrhage, ARF or retinal detachment. We lost two of our cases due to intracerebral haemorrhage and had one case of retinal detachment, 2.8\% HELLP syndrome, 1.4\% ARDS and 2.8\% cases of ARF.

Foetal complications associated with HDP especially pre eclampsia and eclampsia are IUGR, oligohydramnios, preterm delivery, non reassuring foetal heart rate patterns during labour, low APGAR scores at birth and NICU admission. Tavassoli et al. ${ }^{11}$ reported IUGR in $27.5 \%$ of the neonates in severe pre-eclampsia group whereas Yucesoy et al. ${ }^{12}$ reported IUGR in $29.4 \%$ and oligo-hydramnios in $7.5 \%$ cases. ${ }^{11,12}$ In another study the rate of very early preterm delivery ( $<32$ weeks) was $7.8 \%$ in $\mathrm{CH}, 5.9 \%$ in $\mathrm{GH}, 21.2 \%$ in PE and $37.2 \%$ in CHPE while it was only $1.2 \%$ in the control group. The rate of SGA was $16.2 \%$ in $\mathrm{CH}, 22.8 \%$ in $\mathrm{GH}, 50.7 \%$ in $\mathrm{PE}, 37.2 \%$ in CHPE and $5 \%$ in controls. ${ }^{18}$ Similar results were observed in our study also.

Delivery is the ultimate cure for pre eclampsia or eclampsia The decision when to deliver is made when the benefits of delivery outweigh those associated with prolonging pregnancy. There are no randomized trials comparing the optimal method of delivery in women with pre eclampsia. According to the recommendations, a plan for vaginal delivery should be considered in all women with severe disease particularly those beyond 30 weeks gestation. Elective Caesarean section may be preferred in cases before 32 weeks with IUGR and oligo-hydramnios. For women with any HDP, vaginal delivery should be considered unless a caesarean section is required for the usual obstetric indications. If vaginal delivery is planned and the cervix is unfavourable, then cervical ripening should be used to increase the chance of a successful vaginal delivery. Antihypertensive treatment should be continued throughout labour and delivery to maintain systolic BP at $<160 \mathrm{mmHg}$ and diastolic $\mathrm{BP}$ at $<110 \mathrm{mmHg}$. The third stage of labour should be actively managed with oxytocin 5 units intravenously or 10 units intramuscularly, particularly in the presence of thrombocytopenia or coagulopathy. Ergometrine should not be given in any form. ${ }^{19}$

When counselling women who have pre-eclampsia or eclampsia, the main questions about long term prognosis are the possibilities of recurrence in a future pregnancy and the possibilities of chronic hypertension later in life. In fact, the probability of recurrence of pre-eclampsia is approximately $30 \%$ and this probability increases inversely with gestational age at which the patient developed the disease. Prevention of pre-eclampsia and eclampsia can be theoretically achieved at primary, secondary or tertiary levels. Primary prevention is equivalent to avoiding the occurrence of the disease- a task that is impossible at this time because of our limited knowledge about the aetiology and tertiary prevention is synonymous with treatment to avoid complications of the disease. For these reasons efforts should be made to focus on secondary prevention that consists of correcting the pathophysiology of the process to avoid the onset of clinical signs and symptoms. ${ }^{13}$

\section{Conclusion}

Hypertensive disorders of pregnancy pose a major health problem in pregnant women. A substantial burden of maternal and perinatal morbidity and mortality is associated with hypertensive disorders of pregnancy, more so in eclampsia and severe pre-eclampsia group as compared to gestational hypertension, chronic hypertension and mild pre-eclampsia. Routine screening for HDP based on measurement of blood pressure among all pregnant women should be practised as recommended by WHO and where resources are available, it is desirable to do urinary protein analysis at every antenatal visit as a complement to routine blood pressure measurement.

\section{Acknowledgments}

None.

\section{Conflicts of interest}

None.

\section{References}

1. Lippincott W \& Wilkins. Hypertensive disorders in pregnancy. In: Barton JR, et al. (Eds.), Manual of Obstetrics. (8th edn) Wolters Kluver Health, Philadelphia, USA. 2014;pp.183-195.

2. Berzan E, Doyle R, Brown CM. Treatment of preeclampsia: current approach and future perspectives. Curr Hypertens Rep. 2014;16(9):473.

3. World Health Organisation. The world health report 2005- Make every mother and child count. World Health Organization, Geneva. 2005.

4. Rosenberg T, Garbers S, Lipkind H, et al. Maternal Obesity and Diabetes as Risk Factors for Adverse Pregnancy Outcomes: Differences among 4 Racial/Ethnic Groups. Am J Public Health. 2005;95(9):1545-1551.

5. Wagner LK. Diagnosis and management of preeclampsia. American Family Physician. 2004;70(12):2317-2324.

6. Osungbade KO, Ige OK. Public health perspectives of preeclampsia in developing countries: Implication for health system strengthening. Journal of Pregnancy. 2011;2011:6.

7. Audibert F, Boucoiran I, An N, et al. Screening for preeclampsia using first-trimester serum markers and uterine artery Doppler in nulliparous women. Am J Obstet Gynecol. 2010;203(4):383.e1-383.e8.

8. Farnklin University. Simple test can help predict and diagnose preeclampsia. Science Daily. 2010.

9. Report of the National High Blood Pressure Education Program Working Group on High Blood Pressure in Pregnancy. Am J Obstet Gynecol. 2000;183(1):S1-S22.

10. Prakash J, Pandey LK, Singh AK, et al. Hypertension in pregnancy: hospital based study. J Assoc Physicians India. 2006;54:273-278.

11. Tavassoli F, Ghasemi M, Ghomian N, et al. Maternal and perinatal outcome in nulliparious women complicated with pregnancy hypertension J Pak Med Assoc. 2010;60(9):707-710.

12. Yücesoy G, Ozkan S, Bodur H, et al. Maternal and perinatal outcome in pregnancies complicated with hypertensive disorder of pregnancy: a seven year experience of a tertiary care center. Arch Gynecol Obstet. 2005;273(1):43-49. 
13. Assis TR, Viana FP, Rassi S. Study on the major maternal risk factors in hypertensive syndromes. Arq Bras Cardiol. 2008;91(1):11-17.

14. Poon LC, Kametas NA, Chelemen T, et al. Maternal risk factors for hypertensive disorders in pregnancy: a multivariate approach. J Hum Hypertens. 2010;24(2):104-110.

15. Suzuki S, Igarashi M. Risk factors for preeclampsia in Japanese twin pregnancies: comparison with those in singleton pregnancies. Arch Gynecol Obstet. 2009;280(3):389-393.

16. Fernando Arias, Amarnath Bhide, Arulkumaran S, et al. Practical guide to High Risk pregnancy and Delivery (3rd edn), Elsevier, New Delhi, India. 2008;pp.584.
17. Sachan R, Patel ML, Sachan P, et al. Outcomes in hypertensive disorders of pregnancy in the North Indian population. International Journal of Women's Health. 2013;5:101-108.

18. Ferrazzani S, Luciano R, Garofalo S, et al. Neonatal outcome in hypertensive disorders of pregnancy. Early Hum Dev. 2011;87(6):445449

19. Laura AM, Helewa M, Moutquin JM, et al. Diagnosis, evaluation and management of the Hypertensive disorders of pregnancy: executive summary. J Obstet Gynaecol Can. 2014;36(5):416-441. 